\title{
Chest Film Demonstrating Reverse Batwing Pulmonary Opacities in a Patient with COVID-19 Pneumonia
}

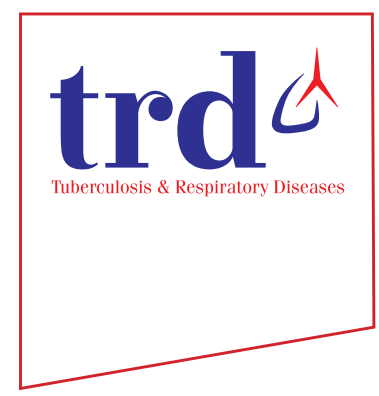

\author{
Chih-Jung Chang, M.D. ${ }^{1}{ }^{\mathbb{D}}$, Sheng-Wei Pan, M.D, Ph.D. ${ }^{1,2}$ and Yuh-Min Chen, M.D., Ph.D. ${ }^{1,2}$ \\ ${ }^{1}$ Department of Chest Medicine, Taipei Veterans General Hospital, Taipei, ${ }^{2}$ School of Medicine, National Yang Ming Chiao Tung \\ University, Taipei, Taiwan
}

A 55-year-old man presented with a 2-day history of fever and shortness of breath. Chest radiography on day 2 revealed a left lower lung infiltrate (Figure 1A). He tested positive for severe acute respiratory syndrome coronavirus 2 by nasopharyngeal swab. He received oxygen therapy via nasal prongs and remdesivir, dexamethasone, and tocilizumab for coronavirus disease 2019 (COVID-19) pneumonia. Chest radiography on day 6 demonstrated newly developed subpleural opacity in the right lung (Figure 1B). The next day, he developed hypoxemia requiring intubation and mechanical ventilation. Arterial blood gases revealed $\mathrm{pH} 7.268, \mathrm{PaO}_{2} 32.5 \mathrm{~mm} \mathrm{Hg}$, and $\mathrm{PaCO}_{2} 53.3 \mathrm{~mm} \mathrm{Hg}$ while using $100 \%$ oxygen. Immediately, he received veno-venous extracorporeal membrane oxygenation (ECMO). A chest film showed profound reverse batwing pulmonary opacities (Figure 1C). Uneventfully, the patient was liberated from ECMO 2 weeks later and ventilator 3 weeks later, when the pulmonary opacities resolved.
Peripheral pulmonary opacities with perihilar region sparing, also known as "photographic negative of pulmonary edema," can be seen in patients with chronic eosinophilic pneumonia, organizing pneumonia, and lung adenocarcinoma ${ }^{1}$. These conditions are characterized by subacute symptoms and poor response to antibiotics. Notably, such a reverse batwing radiographic pattern may present in patients with COVID-19 pneumonia ${ }^{2,3}$, a rapidly progressive disease that has caused 4.8 million deaths since December $2019^{4}$. Alarmingly, 20\% of COVID-19 cases have required hospitalization; of them, $33 \%$ developed acute respiratory distress syndrome ${ }^{5}$. The peripheral and lower-zone distribution of pulmonary infiltrates, one of the typical radiographic findings of COVID-19 pneumonia, might reflect the vulnerabilities of bronchioles and alveoli to virus-induced inflammation ${ }^{6-8}$. Although computed tomography can be a sensitive tool for finding COVID-19 pneumonia ${ }^{8}$, chest radiography is still irreplaceable in
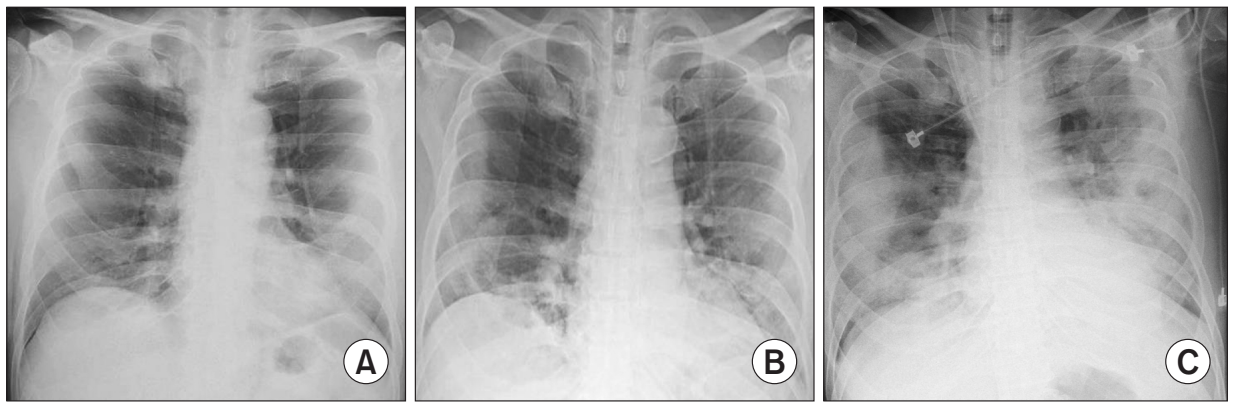

Figure 1. Chest films in a patient with coronavirus disease 2019 pneumonia showed a left lower-lung infiltrate on day 2 (A), demonstrated a new area of subpleural opacity in the right lung on day 6 (B), and disclosed profound reverse batwing pulmonary opacities on day 7 (C).

Address for correspondence: Sheng-Wei Pan, M.D., Ph.D.

Department of Chest Medicine, Taipei Veterans General Hospital, No. 201, Sec. 2, Shih-Pai Rd., Taipei, Taiwan

Phone: 886-2-28757456, Fax: 886-2-28757610, E-mail: sanweipan@gmail.com

Received: Sep. 28, 2021, Revised: Oct. 11, 2021, Accepted: Oct. 16, 2021, Published online: Oct. 20, 2021

@(t) is identical to the Creative Commons Attribution Non-Commercial License (http://creativecommons.org/licenses/by-nc/4.0/). 
screening for COVID-19 in resource-limited areas. In typical clinical presentation and epidemiologic features, a chest film showing reverse batwing changes may alert physicians to the diagnosis of COVID-19 pneumonia during the pandemic period.

\section{Authors' Contributions}

Conceptualization: Chang CJ, Pan SW, Chen YM. Formal analysis: Chang CJ, Pan SW, Chen YM. Writing - original draft preparation: Chang CJ, Pan SW, Chen YM. Writing - review and editing: Chang CJ, Pan SW. Approval of final manuscript: all authors.

\section{Conflicts of Interest}

No potential conflict of interest relevant to this article was reported.

\section{Funding}

No funding to declare.

\section{References}

1. Chaddha U, Lee C. Subacute respiratory illness with peripheral pulmonary opacities. Ann Am Thorac Soc 2018;15:107-9.

2. Ghosh S, Nandolia KK, Tale S, Mrudula K, Soibam PM, Vinay G. Reverse Batwing sign in COVID-19 pneumonia. QJM 2020;113:836.

3. Arora N, Kumar HM. 'The photographic negative of pulmonary oedema' in COVID-19 pneumonia. Postgrad Med J 2021;97:401.

4. WHO coronavirus (COVID-19) dashboard [Internet]. Geneva: World Health Organization; 2021 [cited 2021 Oct 10]. Available from: https://covid19.who.int.

5. Tzotzos SJ, Fischer B, Fischer H, Zeitlinger M. Incidence of ARDS and outcomes in hospitalized patients with COVID-19: a global literature survey. Crit Care 2020;24:516.

6. Zhou S, Zhu T, Wang Y, Xia L. Imaging features and evolution on CT in 100 COVID-19 pneumonia patients in Wuhan, China. Eur Radiol 2020;30:5446-54.

7. Yang W, Sirajuddin A, Zhang X, Liu G, Teng Z, Zhao S, et al. The role of imaging in 2019 novel coronavirus pneumonia (COVID-19). Eur Radiol 2020;30:4874-82.

8. Fang Y, Zhang H, Xie J, Lin M, Ying L, Pang P, et al. Sensitivity of chest CT for COVID-19: comparison to RT-PCR. Radiology 2020;296:E115-7. 